\title{
TRAITEMENTS DE L'INFERTILITE MASCULINE PAR LES TECHNIQUES DE MICROMANIPULATIONS OVOCYTAIRES : REVUE DE LA LITTERATURE
}

\author{
A. Demoulin \\ Centre de Procréation Médicalement Assistée. Université de Liège \\ CHR Citadelle, 1, Bd du 12è de Ligne, 4000 Liège, Belgique
}

\section{TREATMENT OF MALE INFERTILI- TY USING OOCYTE MICROMANIPU- LATION TECHNIQUES : A LITTERATURE REVIEW. During the} last few years assisted reproductive technology has seen the introduction of oocyte micromanipulation and sperm microinjection as therapeutic approaches for severe male factor infertility. However, a review of the international literature has revealed that less than 100 pregnancies have been obtained using such techniques. Fertilization rates are extremely variable and influenced by the strictness of the various authors' criteria for selecting cases. It is concluded that partial zona dissection and zona drilling are less useful than subzonal insemination and intracytoplasmic insemination. Key words : Male infertility, Micromanipulation, Microinjection, PZD, SUZI, ICSI, IVF. Andrologie, 1992, 2 : 61-67.

Lorsqu'une infertilité conjugale trouve son étiologie dans un problème d'altération plus ou moins dramatique du spermogramme, les thérapeutiques, qu'elles soient médicales ou chirurgicales, sont souvent inefficaces. Des palliatifs sont alors proposés aux patients désireux de procréer: le recours à un donneur de sperme, l'insémination artificielle avec ou sans préparation du sperme ou, plus récemment, la fécondation in vitro (FIV). La réduction du nombre de spermatozoïdes nécessaires à la fécondation des ovocytes in vitro laissait augurer d'une solution à la majorité des infertilités masculines. Ce présage ne s'est malheureusement pas confirmé et nombre de spermes, généralement de qualité médiocre, restent inféconds en FIV. Un nouvel espoir est apparu, au cours de ces dernières années, suite à l'introduction des techniques de micromanipulation ovocytaires. Celles-ci facilitent le contact entre les gamètes en réduisant le nombre d'étapes préalables à la pénétration du spermatozoìde dans le cytoplasme ovocytaire.

Le but de cette revue est de décrire les techniques de micromanipulations disponibles et de répertorier, parmi les données de la littérature internationale, les taux de succès obtenus. Les résultats colligés dans cette monographie ont été obtenus dans l'espèce humaine. Les expérimentations animales ont été volontairement éludées bien qu'elles aient constitué le préalable indispensable aux travaux actuels. Les résultats présentés ne concernent que des séries publiées, toutes communications personnelles ou présentations lors de symposium ont été volontairement omises.

A ce jour, il reste cependant à déterminer si ces méthodologies constitueront la panacée pour l'ensemble des problèmes de stérilité masculine qualifiée d'incurable.

\section{METHODOLOGIES Principes}

Les indications masculines de la fécondation in vitro ne constituent qu'une parmi les multiples applications des techniques de micromanipulations dans le domaine de la reproduction. En effet, ces technologies peuvent être utilisée à d'autres fins qui ne seront pas ou peu abordées dans ce travail, telles la fracture de la zone pellucide pour favoriser l'éclosion de l'embryon et l'implantation, l'exérèse de pronoyaux excédentaires, la biopsie embryonnaire pour évaluation diagnostique, ou l'injection de matériel génétique.

Trois méthodes ont été décrites pour faciliter la fécondation des ovocytes par des spermatozoïdes déficients

\section{1) la microperforation de la membrane pellucide}

La méthode consiste à perforer, en un ou plusieurs sites, la membrane pellucide dans le but de faciliter l'entrée des spermatozoïdes dans l'espace périvitellin. Plusieurs techniques ont été décrites : les unes utilisent des agents chimiques, enzymatiques, ou un faisceau laser (zona drilling) les autres résultent d'une action mécanique (zona cutting, zona cracking ou Partial Zona Dissection (PZD).

\section{2) la micro-insémination dans l'espace périvitellin}

Un ou quelques spermatozoïde(s) sont injectés à l'aide d'une micropipette entre la membrane cytoplasmique et la zone pellucide (MIST: Micro-Insemination Sperm Transfert; SUZI: Sub Zonal Insemination).

\section{3) la micro-injection intracytoplasmique}

Un spermatozoïde est injecté dans le cytoplasme de l'ovocyte; il ne reste plus à son noyau qu'à se décondenser (MIMIC: MicroInsemination MicroInjection into Cytoplasm; ICSI: IntraCytoplasmic Sperm Injection)

\section{Matériel nécessaire}

Les ovocytes soumis à une micromanipulation sont observés à l'aide d'un microscope inversé équipé d'optique de type Nomarski. Idéalement, le microscope sera placé dans une enceinte gazée à température constante. Les ovocytes sont manipulés à l'aide de 2 micromanipulateurs hydrauliques ou pneumatiques sur lesquels sont adaptés les différents microinstruments. Ces derniers sont préparés à partir de tubes capillaires en verre. La fabrication de micropipettes mousses, courbées ou biseautées, de micro-aiguilles et de microscalpels nécessite l'acquisition d'une étireuse, d'une microforge et d'une micro affuteuse. Le soin apporté à la fabrication de ces instruments est le garant du succès des étapes ultérieures du processus. Un laser pourrait être également requis si cette méthode se révèle dans l'avenir performante.

\section{Modalités techniques}

Les principes de la FIV, les thérapeutiques de stimulation de la croissance des follicules ovariens, la surveillance du traitement, les techniques de ponction ainsi que les modalités de culture ne seront évidemment pas rappelés dans ce travail.

\section{Isolement de l'ovocyte}

Cette opération, préalable à la micromanipulation de l'ovocyte, est réalisée, selon les auteurs, entre 2 et 8 heures après la ponction (35). Seuls les ovocytes ayant atteint le stade métaphase II feront l'objet d'une micromanipulation. Le cumulus oophorus et la corona radiata sont éliminés par traitement du complexe à l'hyaluronidase (type VIII, 1 à $2 \mathrm{mg} / \mathrm{ml}$ ) diluée dans un milieu de culture exempt de sérum. Ce traitement, d'une durée de 2 à 6 minutes, est suivi d'un pipettage itératif au moyen d'une minipipette (diamètre 100 à $120 \mu \mathrm{m}$ ), qui permet d'éliminer les cellules restantes. Après plusieurs lavages, les ovocytes, entourés de leur zone pellucide, sont à nouveau incubés dans leur milieu de culture classique 
jusqu'au moment de la micromanipulation.

2. Microperforation de la membrane pellucide

a) Techniques chimiques ou enzymatiques (Zona Drilling) :

A l'aide d'une micropipette, quelques nanolitres d'une solution de tyrode acide $(\mathrm{pH} 2.3$. 2.5) sont déposés au contact de la zone pellucide $(20,37)$. Les glycoprotéines sont digérées par ce traitement, entraînant l'apparition d'orifices par lesquels les spermatozoïdes peuvent pénétrer.

Une digestion enzymatique de la membrane à la chymotrypsine $(20,22)$, à la cytochalasine D (42) ou à d'autres enzymes a été également proposée. Ces enzymes fragilisent la zone pellucide qui peut être secondairement perforée mécaniquement.

b) Techniques mécaniques (Partial Zona Dissection, PZD) :

La dissection partielle de la zone pellucide a été, pour la première fois, appliquée à l'espèce humaine par Cohen et son groupe en 1988 (10). La plupart des équipes utilisant cette technique incubent l'ovocyte dans un milieu hyperosmotique contenant du sucrose $(0.1-0.2 \mathrm{M})$ afin d'entraîner une réduction du volume ovocytaire et augmenter ainsi l'espace périvitellin (11). Le risque de perforation de la membrane cytoplasmique lors de la micromanipulation est donc réduit. Cependant, les équipes bien entrainées ont tendance actuellement à omettre cette étape qui ne serait pas dépourvue de toxicité pour l'ovocyte. Placé dans une goutte de $100 \mu \mathrm{l}$ de solution phosphate tamponnée couverte d'huile de paraffine, l'ovocyte est fixé par succion à l'extrémité d'une micropipette dite de maintien (diamètre interne 20 à $30 \mu \mathrm{m}$ ). A l'aide d'une microaiguille, la membrane pellucide est perforée à 1 et 11 heures par simple force mécanique. D'autres auteurs pratiquent une déchirure partielle de la membrane. L'ovocyte est alors remis dans son milieu de culture classique. Un retrait progressif du sucrose entraînerait une augmentation du taux de succès (24). La durée de la manipulation devrait être réduite au minimum et ne pas dépasser quelques minutes. Les variations thermiques et de $\mathrm{pH}$ paraissent également extrêmement délétères à la survie des ovocytes.

\section{c) Techniques au laser:}

L'utilisation du laser pour pratiquer des perforations dans la zone pellucide semble prometteuse. Des résultats préliminaires chez la souris (47) ont été publiés ainsi qu'une revue de la littérature (60). Utilisant un erbium YAG laser (longueur d'onde $2900 \mathrm{~nm}$, diamètre $20 \mu \mathrm{m}$ ), les premiers succes ont été obtenus dans l'espèce humaine (16).
3. Micro-insémination périvitelline (SubZonal Insemination, SUZI)

Comme décrit dans la technique précédente, l'ovocyte, prétraité ou non au sucrose, est fixé à l'aide d'une micropipette dont l'extrémité a été émoussée. Avec l'autre micromanipulateur, une micropipette (diamètre interne 5 à $10 \mu \mathrm{m}$ ), dont l'extrémité a été biseautée $\left(25^{\circ}\right.$ à $\left.35^{\circ}\right)$ et préalablement chargée avec 1 à 7 spermatozoïdes, est introduite dans l'espace périvitellin. Le ou les spermatozoïdes sont alors injectés, ou mieux, on les laisse migrer, dans cet espace.

L'aiguille est ensuite retirée et l'ovocyte transféré dans l'incubateur. Cette manipulation ne doit à nouveau prendre que quelques minutes. Cette technique a été appliquée dans l'espèce humaine pour la première fois en 1987 par LawsKing et al. (34) sans toutefois qu'ils procèdent au transfert des embryons.

\section{Micro-insémination intravitelline} (Intracytoplasmic Sperm Injection, ICSI)

Cette technique, qui consiste à injecter, à l'aide d'une micropipette, une tête de spermatozoïde dans l'ooplasme, n'est encore qu'à ses premiers balbutiements dans l'espèce humaine $(43,63)$. Les premières grossesses après ICSI ont été obtenues par Palermo et al., 1992(49).

\section{Préparation des spermatozoïdes}

Différentes techniques de préparation du sperme sont utilisées. Elles dépendent de la qualité de l'éjaculat. Après lavage et centrifugation, les spermatozoïdes mobiles sont récoltés par migration passive, par sédimentation passive ou après passage sur filtre, généralement un gradient de Percoll. Les techniques de filtration des spermatozoïdes ont actuellement tendance à se généraliser.

Il semble qu'il soit souhaitable d'augmenter la durée d'incubation (pouvant aller jusqu'à 8 heures) avant de pratiquer l'insémination. Certaines équipes collectent même le sperme la veille de la ponction (48). En effet, la capacitation et surtout la réaction acrosomiale nécessitent plusieurs heures, et une partie seulement des spermatozoides accomplira cette réaction. Les quelques spermatozoïdes injectés sous la zone pellucide, ou le spermatozoide introduit dans l'ovocyte, seront néanmoins choisis arbitrairement car la sélection ne pourra s'opérer que sur des critères visibles en microscopie optique, tels que la mobilité et la morphologie. C'est pour cette raison que différentes techniques d'amélioration et de synchronisation de la réaction acrosomiale ont été suggérées $(18,34)$, telles l'addition de médiateurs chimiquement définis (pentoxyfilline, déoxyadénosine...) ou l'incubation dans des fluides biologiques (fluide folliculaire, liquide péritonéal, liquide amniotique...)(19,34). La réaction acrosomiale est également accélérée par la présence de la zone pellucide et des cellules du cumulus oophorus. L'incubation des spermatozoides en présence d'extraits soluble, de la zone pellucide, ou dans un milieu de culture conditionnés de cellules du cumulus, conduit à une augmentation significative de la réaction acrosomique $(13,57,59)$. L'action d'un champ électrique sur les spermatozoides (électroporation) a également été testée avec succès (48).

\section{RESULTATS}

Lorsque nous clôturons cette étude bibliographique, c'est-à-dire en juillet 1992, moins d'une cinquantaine de publications concernant les micromanipulations dans l'espèce humaine ont été recensées dans la littérature française et anglaise. Ces travaux émanent pour la majorité d'un nombre réduit d'équipes. L'analyse des résultats est parfois difficile car certaines données manquent, comme le pouvoir fécondant des spermatozoides lors des tentatives antérieures, l'origine des embryons transférés, les taux d'implantation, les caractéristiques du sperme, etc. De plus, certaines équipes publient à plusieurs reprises les même résultats ou annexent des résultats qui ont fait l'objet de publications antérieures. L'ensemble des résultats publiés est rassemblé dans les tableaux 1 à 3 .

\section{Zona drilling}

Aucune des équipes ayant utilisé les techniques chimiques ou enzymatiques de perforation de la zone pellucide n'a obtenu de grossesse. Les taux de fécondation monospermique variaient entre 15 et $50 \%(15,22)$. Alors qu'elle avait permis d'obtenir d'excellents résultats chez la souris, l'utilisation du zona drilling dans l'espèce humaine s'est rapidement révélée inapplicable suite à la toxicité des substances utilisées sur l'ovocyte humain (37), et ce malgré l'optimisme d'Edirisinghe et al., 1991 (15). Gordon et al. 1988 (22) obtinrent $33 \%$ de lésions ovocytaires, $32 \%$ de fécondation dont $50 \%$ de polyspermie. Ces résultats désastreux ont été confirmés ultérieurement par le même groupe (20) qui avait alors comparé le zona drilling au Tyrode acide ou à la chymotrypsine, et le PZD (tableau 1). Par contre, la technique a été utilisée avec succès

Tableau 1

ZONA DRILLING vs PZD (d'après Garrisi et Coll. 20)

\begin{tabular}{lccccc}
\hline \multicolumn{5}{c}{ FECONDATION $(\%)$} \\
\hline & Cycles & $\begin{array}{c}\text { FIV } \\
\text { classique }\end{array}$ & $\begin{array}{c}\text { Micro- } \\
\text { manipulation }\end{array}$ & $\begin{array}{c}\text { Poly- } \\
\text { spermie }\end{array}$ & Clivage \\
\hline Tyrode Acide & 10 & 11,5 & 17,5 & 42,8 & 100 \\
Chymotrypsine & 9 & 6,5 & 25 & 42,8 & 58,3 \\
PZD & 16 & 100 & 21,7 & 16,1 & 61
\end{tabular}


Tableau 2 : Résultats obtenus en PZD

\begin{tabular}{|c|c|c|c|c|c|c|c|c|c|c|c|}
\hline Autcurs & Réf. & $\begin{array}{l}\text { Nb de } \\
\text { cycles } \\
\text { traités }\end{array}$ & $\begin{array}{l}\text { Nbre } \\
\text { de } \\
\text { pat. }\end{array}$ & $\begin{array}{l}\text { Nbre embr. } \\
\text { par ovo } \\
\text { microm. }\end{array}$ & $\begin{array}{c}\text { Nh embr. } \\
\text { par ovo } \\
\text { non microm. }\end{array}$ & $\begin{array}{l}\text { Qualité } \\
\text { Sperme } \\
\text { X } 10.6\end{array}$ & $\begin{array}{l}\text { Gross. } \\
\text { Transf. } \\
\text { microm. }\end{array}$ & $\begin{array}{l}\text { Gross. } \\
\text { Transt: } \\
\text { mixte }\end{array}$ & $\begin{array}{c}\text { Gross. évol. } \\
\text { Transf. } \\
\text { microm. }\end{array}$ & $\begin{array}{c}\text { Fréq. } \\
\text { polyspermie }\end{array}$ & $\begin{array}{l}\text { Taux } \\
\text { d' implant. }\end{array}$ \\
\hline Malter et Cohen 1989 & 37 & 11 & 11 & $27 / 35$ & $16 / 32$ & OAS & $3 / 7$ & & $3 / 7$ & $4 / 27$ & $4 / 13$ \\
\hline Coben et al. 1989 & 11 & 18 & 18 & $42 / 55$ & $28 / 55$ & OAS & $1 / 5$ & $4 / 9$ & $1 / 5$ & $11 / 42$ & $?$ \\
\hline Garrisi et al. 1990 & 20 & 16 & 15 & $31 / 143$ & $4 / 102$ & & $0 / 9$ & & & $5 / 31$ & $0 ?$ \\
\hline Jean el al. $1990^{\circ}$ & 26 & 5 & 58 & $0 / 25$ & $1 / 30$ & $15-40$ & $0 / 0$ & & & $?$ & $0 ?$ \\
\hline Chang et al. 1991 & 5 & 1 & 1 & $2 / 2$ & $2 / 3$ & & $1 / 1$ & & $1 / 1$ & $0 / 2$ & $1 / 2$ \\
\hline Coher et al. 1991 & 12 & 61 & 6] & $82 / 293$ & $31 / 128$ & OAS & $3 / 21$ & $2 / 6$ & $?$ & $?$ & $?$ \\
\hline Cohen et al. 1991 & 7 & 57 & 57 & $97 / 267$ & 681196 & OAS & $4 / 21$ & $8 / 17$ & ? & $23 / 97$ & $16 / 95$ \\
\hline Hill ct al. 1991 & 24 & 16 & 14 & $22 / 88$ & $16 / 65$ & $1 / 200$ & $0 / 5$ & $1 / 3$ & & $5 / 22$ & $0 ?$ \\
\hline Payne et al. 1991 & 50 & 22 & 23 & $47 / 147$ & $25 / 75$ & OAS & $0 / 15$ & & & $2 / 47$ & $0 ?$ ? \\
\hline Simon et al. 1991 & 58 & 22 & 22 & $39 / 15$ & $3 / 55$ & $3-110$ & $0 / 9$ & & & $5 / 39$ & $0 ?$ \\
\hline Tucker el al. 1991 & 61 & 70 & 67 & $73 / 28$ & $33 / 254$ & $0.6-7.5$ & $6 / 17$ & $10 / 13$ & $2 / 17$ & $31 / 73$ & $6 / 27$ \\
\hline Barlow et aj. 1942 & 2 & 28 & $?$ & $37 / 171$ & $3 / 105$ & & $3 / 13$ & & $2 / 3$ & $7 / 37$ & $?$ \\
\hline Jean et al. 1992 & 25 & 20 & 20 & $15 / 86$ & $3 / 100$ & $5-\mathrm{N}$ & $1 / 3$ & & $0 / 3$ & $10 / 15$ & $?$ \\
\hline Van der Zwalmen ct al. 1992 & 62 & 43 & 34 & $51 / 254$ & $6 / 111$ & $12-\mathrm{N}$ & $3 / 20$ & $0 / 3$ & $2 / 20$ & 10/5] & $3 / 39$ \\
\hline
\end{tabular}

pour faciliter l'éclosion des embryons et leur implantation secondaire (8). Cette technique parait efficace surtout lorsque la zone pellucide est épaisse (plus de $15 \mu \mathrm{m}$ ), et chez les patientes agées de plus de 38 ans. Le zona drilling serait préférable à la dissection mécanique introduite antérieurement par le même groupe (9). Il semblerait donc que la solution acide soit moins nocive pour les embryons qu'elle ne l'était pour les ovocytes avant fécondation.

\section{Partial zona dissection}

Le tableau 2 rassemble l'ensemble des résultats publiés après PZD. L'importance des variations observées peut s'expliquer par des choix de populations différentes. Ainsi, dans l'étude de Tucker et al., 1991 (61), portant sur 67 couples, 14 seulement avaient présenté un échec de fécondation lors d'une tentative antérieure. Sur les 70 cycles étudiés, 16 ont montré un taux de fécondation identique en FIV conventionnelle et en PZD, 35 n'ont fécondé qu'en PZD et les 19 tentatives restantes se sont soldées par un échec complet de fécondation dans les deux techniques. Dans une autre publication,(7), la même équipe montre que les résultats obtenus en PZD et en FIV classique sont identiques (34 et $36 \%$ ). Si par contre, seuls les couples ayant présenté un échec de FIV dans leurs antécédents sont sélectionnés, les taux de fécondation sont respectivement de 14 et $32 \%$ et la différence devient significative. Lorsqu'une sélection plus sévère des cas est opérée,on constate que la PZD permet une amélioration des taux de fécondation ( $20 \%$ versus $5,4 \%$ ) (62).

L'étude approfondie du tableau 2 montre qu'à l'exception des cas isolés, les publications de séries plus ou moins larges émanent de 6 équipes différentes (références les plus récentes: $24,25,50,58,61$ et 62 ). Elles portent sur un total de 193 cycles et 1007 ovocytes soumis à la PZD. Les taux de fécondation globaux sont respectivement de 24,5 et de $13,0 \%$ selon le type d'insémination: PZD ou FIV classique. La fréquence de la polyspermie est de $25,5 \%$. Un transfert d'embryons résultant de la micromanipulation a pu être réalisé chez 69 patientes (35,8\%). Dix grossesses ont été diagnostiquées dont 4 ont évolué, soit 2,1\% de grossesse évolutive par cycle pris en charge. Par contre les transferts mixtes, c'est à dire un mélange d'embryons résultant des PZD et de l'insémination classique, ont permis, l'obtention de 11 grossesses sur 19 transferts $(58 \%)$.

\section{Subzonal insemination}

Les résultats publiés après micro-injection dans la zone périvitelline sont rassemblés dans le tableau 3. Dans l'étude de Fishel et al., 1992 (19) qui collige d'ailleurs les résultats de leurs travaux antérieurs $(17,18), 36 \%$ des patientes ont eu au moins 1 ovocyte fécondé. Sur 114 patientes, les taux de fécondation en FIV classique et après SUZI étaient respectivement de 6 et $39 \%$ ( $p$ significatif). Ces résultats sont surprenants et en contradiction avec ceux publiés par les mêmes auteurs (17), où ils observaient des taux de féconcatif). Lorsque les SUZI sont pratiquées chez des patientes ayant eu au moins 2 échecs de fécondation, et que la moitié des ovocytes est inséminée classiquement, et l'autre moitié est micromanipulée, il n'y a aucune fécondation dans le groupe témoin alors que $19.6 \%$ des ovocytes sont fécondés après microinjection (65). Actuellement ils dation respectivement de 7,6 et $14,5 \%$ (p signifi-

Tableau 3: Résultats obtenus en SUZI

\begin{tabular}{|c|c|c|c|c|c|c|c|c|c|c|c|c|}
\hline Auteurs & Réí. & $\begin{array}{l}\text { Nb de } \\
\text { cycles } \\
\text { Iraités }\end{array}$ & $\begin{array}{l}\text { Nhre } \\
\text { de } \\
\text { pat. }\end{array}$ & $\begin{array}{c}\text { Nbre embr. } \\
\text { par ovo } \\
\text { microm. }\end{array}$ & $\begin{array}{l}\text { Nb embr. } \\
\text { par ovo } \\
\text { non microm. }\end{array}$ & $\begin{array}{l}\text { Qualité } \\
\text { Sperme } \\
\text { X10.6 }\end{array}$ & $\begin{array}{l}\text { Gross. } \\
\text { Transf. } \\
\text { microm. }\end{array}$ & $\begin{array}{l}\text { Gross. } \\
\text { Transf. } \\
\text { mixle }\end{array}$ & $\begin{array}{l}\text { Gross. Evol. } \\
\text { Transf. } \\
\text { microm. }\end{array}$ & $\begin{array}{l}\text { Fréq. } \\
\text { polyspermie }\end{array}$ & $\begin{array}{l}\text { NB SPZ } \\
\text { injectés }\end{array}$ & $\begin{array}{c}\text { Taux } \\
\text { dimplant. }\end{array}$ \\
\hline Bongso et al. 1989 & 4 & 1 & 1 & $3 / 3$ & & $87(0 \%)$ & $0 / 1$ & & & $0 / 3$ & 1 out & $0 / 3$ \\
\hline Fishel et al. 1990 & 18 & 85 & 85 & $55 / 369$ & $6 / 112$ & $1.5-30$ & $3 / 31$ & $?$ & $3 / 31$ & $2 / 55$ & $3.4 /-2.1$ & $4 / 47$ \\
\hline Olar et al. 1990 & 46 & 1 & 1 & $3 / 6$ & $0 / 6$ & P. Epidi. & $0 / 1$ & & & $0 / 3$ & 1 & $0 / 3$ \\
\hline Wolf el Jouannet 1991 & 65 & 17 & 11 & 28191 & $0 / 73$ & OAS et $N$ & $2 / 9$ & & ? & $10 / 28$ & ? & $?$ \\
\hline Cohen et al. 199 ! & 12 & 19 & 19 & 28187 & & OATS & $0 / 10$ & & & $14 / 28$ & $1-12$ & a? \\
\hline Cohen et al. 199 & 7 & 47 & 47 & $65 / 254$ & $22 / 71$ & OAS & $5 / ?$ & & $?$ & $13 / 65$ & & $?$ \\
\hline Fishul et al. 1991 & 17 & 130 & 130 & 691539 & $19 / 250$ & OATS & $6 / 39$ & l? & 4139 & $?$ & $3.7+/-2.1$ & $9 / 61$ \\
\hline \multirow{2}{*}{ Ng et al. 1991} & 43 & 131 & 131 & $143 / 771$ & $15 / 95$ & 0.1 .86 & $5 / 58$ & & $1 / 58$ & $15 / 143$ & $+1-7$ & $?$ \\
\hline & & 35 & 35 & $35 / 188$ & & OAS et $\mathrm{N}$ & $0 / 2$ & & & $7 / 35$ & $+1-7$ & ? \\
\hline Wittemer el al. 1991 & 64 & 1 & 1 & $1 / 10$ & & $52 \times 10.6$ & {$[/]$} & & $1 / 1$ & $0 / 1$ & $\Rightarrow 10$ & $1 / 1$ \\
\hline Fishel et at. 1992 & 19 & 225 & 225 & $150 / 1003$ & $22 / 364$ & OAS et $\mathrm{N}$ & $12 / 82$ & $?$ & $?$ & $7 / 150$ & $4.9+t-0.1$ & $?$ \\
\hline Sakkas ef al. 1992 & 52 & 150 & 139 & $134 / 942$ & & OAS & $6 / 58$ & & $2 / 58$ & $19 / 134$ & $1-4$ & ? \\
\hline Palermo et al. 1992 & 48 & 44 & 43 & $134 / 464$ & & $0,9-158$ & $7 / 34$ & & $4 / 7$ & $13 / 34$ & $\Rightarrow 3$ & ? \\
\hline
\end{tabular}

*Microinsémination à $\mathrm{J}+\mathrm{l}$ pour échec de fécondation

$0,9-15$ ont obtenu 5 grossesses sur 24 transferts.

Quelques auteurs ont rapporté des succès isolés. Ainsi, des fécondations ont-elles été obtenues en SUZI après injection de spermatozoïdes immobiles (4), ou de spermatozoïdes épididymaires (46).

Lorsqu'on compare l'efficacité de la PZD et de la SUZI chez 19 patients lors d'une même tentative de FIV, des taux de fécondation similaires (26 et $32 \%$ ) ainsi qu'une fréquence de polyspermie identique (42 et $50 \%$ ) sont décrits (12). La même année, la même équipe publiait une étude portant sur 47 couples réalisant une tentative de FIV pour indication masculine; les taux de fécondation étaient respectivement de $31 \%$ (22/71), $21 \% \quad(31 / 148)$ et de $26 \%(65 / 254)$ en FIV classique, en PZD et en SUZI (7).

Si le taux de fécondation s'améliore lorsqu'on augmente le nombre de spermatozoïdes injectés, on observe en corollaire une fréquence de polyspermie accrue (32), pouvant atteindre $100 \%$ des ovocytes lorsque $\geq 8$ spermatozoïdes sont déposés dans l'espace périvitellin (42).

Un examen attentif du tableau 3 révèle que 6 équipes seulement ont publié des résultats sur des séries de patients traités par la SUZI (références: $7,19,43,48,52$ et 65 ). Sur 614 cycles pris en charge, 3525 ovocytes ont été microinjectés alors que 603 ont été inséminés classiquement. Les taux de fécondation sont respectivement dans les deux groupes de 18,5 et $9,8 \%$. Les fécondations polyspermiques sont de $11,8 \%$ après SUZI. Sur 241 transferts $(39,3 \%), 32$ patientes ont eu un test de grossesse positif $(13,2 \%)$. La publication de Cohen et al, 1991 (7) ne permet pas de déterminer l'origine des embryons dans les 5 grossesses obtenues. Le nombre de grossesses évolutives n'a pas été précisé par la plupart des auteurs.

\section{Intracytoplasmic injection}

Seuls des résultats préliminaires ont été publiés à ce jour. Lanzendorf et al., 1988 (31) ont inséminé 20 ovocytes maturés in vitro et ont obtenu 6 embryons à 2 PN qui ne furent pas transférés. Veeck et al., 1989 (63) ont injecté un spermatozoide dans le cytoplasme de 85 ovecytes; $24 \%$ se fécondèrent normalement ( $2 \mathrm{PN})$, $13 \%$ se divisèrent, il fut procédé à 7 transferts sans obtenir de grossesse. Ng et al., 1991 (43) ont injecté 38 ovocytes obtenus chez 3 patientes différentes; 12 ovocytes ne survécurent pas à la manipulation, 4 étaient au stade $2 \mathrm{PN}$ et ont été transférés chez 2 patientes différentes sans qu'il en résulte de grossesse. Et enfin, Palermo et al., 1992 rapportent 4 grossesses sur 60 patientes 
traitées $(6,7 \%) ; 133$ ovocytes avaient été fécondés sur 234 micromanipulés $(57 \%) ; 17$ patientes avaient bénéficié d'un transfert (28\%).

\section{DISCUSSION}

En préambule,signalons l'existence d'une excellente revue de la littérature sur l'ensemble des applications des micromanipulations à l'espèce humaine, publiée en 1990 (45).

La réussite d'une fécondation tant in vivo qu'in vitro est conditionnée par la maturité et la qualité de l'ovocyte d'une part et, l'intégrité des propriétés fonctionnelles et cinétiques des spermatozoïdes d'autre part. Ainsi, lorsque les spermatozoides arrivent au contact du complexe cumulus-ovocyte, il leur reste de nombreuses étapes à franchir: traversée du cumulus et de la corona radiata, fixation à la zone pellucide, perforation de cette dernière, pénétration de la membrane cytoplasmique, blocage de la polyspermie, décondensation nucléaire et formation du pronoyau masculin, et enfin fusion de ce dernier avec le pronoyau féminin. Pour que les spermatozoides soient capables d'effectuer ces différentes étapes préalables et contemporaines, à la fécondation, ils doivent subir une capacitation et la réaction acrosomiale doit s'accomplir $(53,54)$. Dans une population de spermes fertiles 6 à $10 \%$ des spermatozoïdes ont fait leur réaction après $3 \mathrm{~h}$. de culture $(35,41)$. In vitro, ce pourcentage peut être augmenté et synchronisé par l'addition de différents médiateurs au milieu de culture et par l'allongement de la période d'incubation. (voir paragraphe préparation des spermatozoides).

Tant in vivo qu'in vitro, les patients, dont le spermogramme est altéré présentent réguliérement une infertilité due à un pouvoir fécondant réduit ou même nul. Une relation directe avec la gravité des altérations a été observée, le facteur le plus discriminant serait pour Acosta et al. (1) la tératospermie. Selon d'autres auteurs, les paramètres numération et mobilité ne seraient pas corrélés avec le pouvoir fécondant après PZD, mais aucun embryon ne s'implanterait lorsque les ovocytes sont fécondés par un sperme contenant moins de 5\% de spermatozoides à forme normale (30).

La présence d'une oligospermie importante s'associe à une absence de fécondation, même après micromanipulation ovocytaire (PZD) (61) et, s'il y a fécondation, l'implantation embryonnaire est perturbée (12). D'autres auteurs ont observé une corrélation entre le taux de fécondation en SUZI et le nombre de spermatozoïdes dans l'éjaculat, confirmant ainsi que les techniques de microinjection n'apporteront pas la solution à tous les problèmes d'oligoasthénospermie (43).
En SUZI, le taux de fécondation est significativement plus faible $(8 \%)$ lorsque le nombre de spermatozoides mobiles par éjaculat est inférieur à 1 million (étude sur 79 cas), et comparé au reste de la population $(\mathrm{n}=131)$ où il y avait plus de 1 million de spermatozoides mobiles par éjaculat ( $24 \%$ de fécondation). Les mêmes variations sont d'ailleurs observées en FIV classique; celle ci étant réalisée sur une autre fraction des ovocytes: moins de 1 million de spermatozoides, $0 \%$ de fécondation; plus de 1 million, 6,6 \%)(19).Un retard de fécondation et/ou un développement embryonnaire anormal sont décrits chez les patients dont le sperme est pathologique (51). Les pathologies nucléaires seraient en ce sens plus graves que les anomalies de l'acrosome (58). Des études animales montrent cependant une absence de relation entre les caractéristiques physiques des spermatozoïdes et leur matériel génétique (40). Il apparait donc qu'une réduction du taux d'implantation et une augmentation de la fréquence des fausses couches s'observent en cas d'oligotératospermie $(1,7,12)$. Cette assertion ne se vérifierait pas lorsque les embryons résulteraient d'une SUZI (7).

Il ressort donc de cette analyse que les paramères classiques du sperme, numération, mobilité et morphologie, s'il n'ont pas une valeur discriminante parfaite, permettent néanmoins de prédire les chances de succès tant d'une FIV classique qu'après micromanipulation. Et force est de constater que les techniques d'injection sous pellucidaire ne constituent pas la panacée, mais permettent seulement de reculer les limites d'acceptation d'un patient. Les résultats disponibles en injection intracytoplasmique sont encore trop fragmentaires pour préjuger de leur valeur et pour affirmer que cette technique résoudra tous les problèmes masculins hormis bien sûr les aplasies germinales complètes.

Deux problèmes majeurs sont apparus rapidement au cours de l'analyse des résultats: certains auteurs ont publié à plusieurs reprises les mêmes séries, et d'autre part, il est souvent difficile de connaître avec précision les critères d'inclusion utilisés.

L'étude - non exhaustive - de la littérature que nous avons réalisée permet d'estimer que le nombre de grossesses en PZD est compris entre 10 et 15 dont 5 à 8 seraient évolutives sur plus ou moins 200 cycles ponctionnés. En SUZI, sur plus de 600 cycles ponctionnés, on compte moins de 40 grossesses. Selon un rapport rassemblant les résultats présentés à 2 congrès (Rome 1990 et Paris 1991) il y aurait eu, jusqu'en juillet 1991, en PZD, 13 grossesses cliniques après transfert d'embryon obtenus après une micromanipulation, soit $11 \%$ par transfert et $4.5 \%$ par cycle, tandis que 16 grossesses ont été obtenues après transferts mixtes (embryons intacts et embryons ayant subi une micromanipulation). En SUZI, 55 grossesses auraient été obtenues sur 333 transferts $(16.5 \%)$ et sur 653 ponctions $(8.4 \%)$. Il n'est pas précisé s'il s'agit de transferts purs ou mixtes, ni les indications des micromanipulations (33). Il faut signaler qu'un certain nombre de ces cas présentés n'ont pas été confirmés dans une publication internationale. Plus récemment, lors de l'International Symposium on Preimplantation Genetics and Assisted Fertilization (Bruxelles février 1992), les résultats d'une enquête menée auprès de différents centres rapporte que les taux de grossesses par cycle étaient respectivement de $3,3,10,1$ et $13,3 \%$ en PZD, SũZI et ICSI (soit 11,49 et 8 grossesses dans chaque groupe).

L' analyse de certains publications montre clairement que les techniques de micromanipulation sont inutiles si les critères de sélection ne sont pas rigoureux. Plusieurs études ont ainsi démontrés que les taux de fécondation étaient similaires en FIV classique et après micromanipulation si ces techniques sont appliquées en première intention. Donc certaines grossesses obtenues après micromanipulation l'aurait également été après FIV conventionnelle. Il parait évident que seuls des cas d'absence répétée de fécondation in vitro devraient être inclus dans des études ayant pour but de démontrer l'efficacité de ces nouvelles technologies. A ce jour, il est donc impossible d'effectuer une méta-analyse qui permettrait d'évaluer l'apport exact de la PZD et/ou de la SUZI dans des échecs répétés de fécondation in vitro.

Selon Fishel et al., 1990 (18), 20\% des infertilités idiopathiques soumises à une tentative de FIV présentent une absence de fécondation avec, pour certaines, une non fixation des spermatozoïdes à la membrane pellucide. Différentes techniques ont donc été utilisées pour tenter d'améliorer le taux de fécondation dans ces cas: augmentation du nombre de spermatozoïdes inséminés, sélection des spermatozoïdes (gradient de Percoll ou de Ficoll, passage sur laine de verre), addition de médiateurs chimiques, retrait du cumulus, culture en microgoutte ou en capillaire, etc. Cependant, il semble que la traversée de la membrane pellucide reste dans de nombreux cas d'infertilité idiopathique l'obstacle majeur à la pénétration des spermatozoïdes dans l'ovocyte. En effet, une dépellucidation complète conduit presque inéluctablement à la fécondation, et souvent à la polyspermie $(3,14)$. En présence de spermatozoides non fécondants lors de tentatives de FIV antérieures, une fécondation a été obser- 
vée dans $70 \%$ des cas après dépellucidation d'ovocytes agés de 48 heures (21). La présence et une intégrité relative de la zone pellucide constjtuent donc une étape limitante dans le processus de la fécondation, mais sont plutôt aussi le garant de la monospermie. Les résultats des micromanipulations le confirment. Les rôles respectifs de l'ovocyte et de la membrane pellucide dans le blocage à la polyspermie ont été évalués, et diffèrent d'ailleurs d'une espèce à l'autre. $(18,38,39$, $45,55,56)$.

Dans l'espèce humaine, et en contraste avec les expérimentations chez la souris (23), l'utilisation de solution de Tyrode acide pour perforer la zone pellucide et faciliter la fécondation n'est pas applicable. La survie, la fécondation et le clivage des ovocytes humains sont extrêmement faibles $(20,22,37)$, car la solution acide perturbe le processus méiotique par arrêt au stade anaphase II (43). De plus, les solutions de tyrode acide induisent une division parthénogénétique des ovocytes (27). Il en serait de même avec les traitements enzymatiques, ainsi la cytocholasine modifierait l'architecture du cytosquelette réduisant le taux de fécondation (42). Cette méthodologie est donc actuellement abandonnée dans cette indication, alors qu'elle pourrait être utile pour favoriser l'éclosion embryonnaire (8).

Des avis contradictoires sont émis concernant les avantages et les inconvénients des micromanipulations pour opérer une sélection des spermatozoides. En SUZI, contrairement à la PZD, la sélection naturelle des spermatozoìdes ayant fait leur réaction acrosomique n'a pas lieu (12). Suite à une sélection arbitraire, en SUZI et plus encore en ICSI, on pouvait craindre une augmentation de la fréquence des anomalies génétiques. En pratique c'est l'inverse qui a été observé (7). De plus les travaux expérimentaux de Kola et al. en 1990 (29) ont apaisé cette inquiétude car aucune augmentation de la fréquence d'aneuploidie n'a été observée après SUZI. Bien que le nombre de grossesses à terme résultant d'une SUZI ou d'une PZD soit encore assez reduit, il ne semble pas que ces techniques augmentent la fréquence des malformations foetales. Par contre, le nombre de grossesses résultant d'ICSI est actuellement trop faible pour conclure à l'innocuité de cette technique. Il ressort de l'expérience de plusieurs auteurs que la SUZI est néanmoins plus efficace que la PZD dans les problèmes d'infertilité masculine. Cette dernière technique devrait rapidement disparaitre de l'arsenal thérapeutique ayant pour but l'amélioration du taux de fécondation.

Deux problèmes sont apparus suite à l'introduction des techniques de micromanipulation: la polyspermie et la survie embryonnaire. En SUZI, il semble exister un parallélisme entre le nombre de spermatozoides déposés dans l'espace périvitellin et le nombre de pronoyaux observé (19, 32). L'insémination par 2 ou 3 spermatozoides réduit ce risque à des valeurs acceptables;par contre, l'augmentation du nombre de spermatozoides injectés n'améliore pas le taux de fécondation monospermique (52). D'autre part, la fréquence des lésions cellulaires dépend de l'habileté de l'expérimentateur. Les chiffres varient entre $3 \%$ (12) et $15 \%$ (18) selon les équipes. De plus les micromanipulations pourraient être responsable d'une éclosion embryonnaire plus précoce avec perturbations des processus immunologiques liés à l'implantation. Dans cette optique, Cohen et al., 1990 (9) ont placé sous couverture corticoide et antibiotique leur patientes dont les embryons avaient subi une micromanipulation pour favoriser l'éclosion. Un taux de grossesse de $28 \%$ a été obtenu chez ces patientes qui avaient présenté des échecs d'implantation lors des tentatives antérieures.

Il parait indéniable que les techniques d'insémination périvitelline et intracytoplasmique constituent des étapes importantes dans le traitement des infertilités masculines. Beaucoup de patients considérés à ce jour comme définitivement stériles peuvent espérer obtenir une grossesse. Il est cependant prématuré de vouloir préciser les limites des indications.Pour juger de l'efficacité des techniques, les couples sélectionnés devraient répondre à certains critères stricts:

$1^{\circ}$ en cas de spermes classiquement normaux ou subnormaux, avoir eu au moins deux échecs de fécondation portant sur un nombre minimum d'ovocytes. Il devrait s'agir d'un critère minimum puisque dans 70 à $80 \%$ des cas, un échec de fécondation lors d'une tentative se solde par une fécondation lors d'une tentative ultérieure $(6$, 21).

$2^{\circ}$ si les caractéristiques du spermogramme ne permettent pas la réalisation d'une tentative de FIV classique (moins de un million de spermatozoides mobiles par éjaculat (19), le SUZI ou l'ICSI peuvent être envisagé d'emblée.

$3^{\circ}$ en cas d'hypofécondances majeures et répétées en FIV classique, l'apport des techniques de micromanipulation, en terme de grossesses évolutives, reste encore à démontrer.

Le recours aux techniques de micromanipulation peut également être d'application dans certaines circonstances particulières et exceptionnelles: ainsi des tentatives de SUZI ont-elles été effectuées avec des spermes congelés avant chimio- ou radio-thérapie, et ayant mal résisté au processus cryopréservatif (28); également après collecte des spermatozoides dans les canaux déférents ou dans l'épididyme. De plus, l'absence complète de mobilité pourrait être court-circuitée par l'utilisation de l'ICSI.

Malheureusement, à ce jour, il n'existe pas de test de sélection dont le pouvoir discriminant soit absolu. Le test de pénétration des ovules de hamster dépellucidés semble garder un intérêt majeur. Des tests homologues pourraient se développer : des ovocytes non fécondés après 24 ou 48 heures sont soit dépellucidés ou micromanipulés, et mis en présence des spermatozoïdes à étudier $(3,14)$. Ces techniques pourraient tester le pouvoir fusiogène des spermatozoïdes et déterminer l'indication au recours à l'injection intraooplasmique.

\section{CONCLUSION}

Les techniques de micromanipulation (PZD, SUZI ou ICSI) n'ont permis a ce jour d'obtenir qu'un nombre limité de grossesses (moins de 100 recensées). Les taux de fécondation restent relativement faibles. Il est donc impératif de poursuivre les travaux de recherche pour, d'une part mieux déterminer les étiologies des infertilités susceptibles de bénéficier de ces techniques, et d'autre part améliorer les modalités méthodologiques afin d'augmenter les taux de fécondation.

Vu les taux de succès actuels, il nous semble logique de continuer à considérer ces techniques comme expérimentales, et de les réserver à quelques centres spécialisés. Avant d'engager un couple dans une telle procédure, l'équipe médicale se doit de le prévenir des chances réelles de réussite.

\section{REFERENCES}

1 - Acosta AA, Oehniger S, Morshedi M, Swanson RJ, Scott R, Irianni F: Assisted reproduction in the diagnosis and treatment of the male factor. Obstet. Gynaecol. Surv., 1988, 44:1-18.

2 - Barlow P, Van der Zwalmen P, Delvigne A, Van Hoeck J, Schoysman R, Leroy F: L'assistance à la fécondation de l'ovocyte humain par micromanipulation. Rev. Med. Brux., 1992, 13:13-19.

3 - Bongso A, Fong CY, Ng SC, Ratman S: Fertilization cleavage and cytogenetics of 48-hour zona-intact and zonafree human unfertilized oocytes reinseminated with donor semen. Fertil. Steril., 1992, $57: 129-133$

4 - Bongso TA, Sathananthan AH, Wong PC, Ratnam SS, Ng SC, Anandakumar C, Ganatra S: Human fertilization by microinjection of immotile spermatozoa. Human Reprod., 1989, 4:175-179.

5 - Chang SY, Soong YK, Chang M, Lin PW, Guu $\mathrm{HF}$, Wang ML: A clinical pregnancy after a simple method of zona cutting, cryopreservation and zygote intrafallopian transfer. Fertil. Steril., 1991, 55:420422. 
6 - Coates TE, Check JH, Choe J, Nowroozi K, Lurie D, Callan C: An evaluation of couples with failure of fertilization in vitro. Human Reprod., 1992, 7:978-981.

7 - Cohen J, Alikani M, Malter HE, Adler A, Talansky BE, Rosenwaks Z: Partial zona dissection or subzonal sperm insertion: microsurgical fertilization alternatives based on evaluation of sperm and embryo morphology. Fertil. Steril,. 1991, 56:696-706.

8 - Cohen J, Alikani M, Trowbridge J, Rosenwaks: Implantation enhancement by selective assisted hatching using zona drilling of human embryos with poor prognosis. Human Reprod., 1992, 7:685-691.

9 - Cohen J, Elsner C, Kort H, Malter H, Massey J, Mager MP, Wiemer K: Impairment of the hatching process following IVF in the human and improvement of implantation by assisting hatching using micromanipulation. Human Reprod., 1990, 5:7-13

10 - Cohen J, Malter H, Fehilly C, Wright G, Elsner C, Kort H, Massey J: Implantation of embryos after partial opening of oocyte zona pellucida to facilitate sperm penetration. Lancet., 1988, 2:162.

11 - Cohen J, Malter H, Wright G, Kort H, Massey J, Mitchell D: Partial zona dissection of human oocytes when failure of zona pellucida penetration is anticipated. Human Reprod., 1989, 4: 435-442.

12 - Cohen J, Talansky BE, Malter H, Alikani M, Adler A, Reing A, Berkeley A, Graf M, Davis O, Liu $\mathrm{H}$, Bedford JM, Rosenwaks Z: Microsurgical fertilization and teratospermia. Human Reprod., 1991, 6:118123.

13 - Cross NL, Morales P, Overstreet JW, Hanson FW: Induction of acrosome reactions by human zona pellucida. Biol. Reprod., 1988, 38:235-244.

14 - Demoulin A, Pignon MR, Gerday C, Jouan C, Dubois M: The use of zona-free human oocytes as a test of fertilizing capacity of human spermatozoa. 1992, En préparation.

15 - Edirisinghe WR, Wales RG, Chapman HM, Yovich JL: Assisted fertilization of mouse oocytes and preleminary results for human oocytes using zona drilling. J. In Vitro Fert. Embryo Transfer., 1991, 8:4855 .

16 - Feichtinger W, Strohmer H, Fuhrberg P, Radivojevic K, Antinori S, Pepe G, et al: Photoablation of oocyte zona pellucida by erbium-yag laser for in vitro fertilisation in severe male infertility. Lancet, 1992, 339:811.

17 - Fishel S, Antinori S, Jackson P, Johnson J, Rinaldi L: Presentation of six pregnancies established by subzonal insemination (SUZI). Human Reprod., 1991, 6:124-130.

18 - Fishel S, Jackson P, Antinori S, Johnson J, Grossi S, Versaci C: Subzonal insemination for the alleviation of infertility. Fertil. Steril., 1990, 54:828-833.

19 - Fishel S, Timson J, Lisi F, Rinaldi L: Evalution of 225 patients undergoing subzonal insemination for the procurement of fertilization in vitro. Fertil. Steril., 1992, 57:840-849.
20 - Garrisi GJ, Talansky BE, Grunfeld L, Sapira V, Navot D, Gordon JW: Clinical evaluation of three approaches to micromanipulation-assisted fertilization. Fertil. Steril., 1990, 54:671-677.

21 - Gerday C, Jouan C, Dubois M, Demoulin A: L'échec de fécondation en FIV: intérêt d'un taux de récolte ovocytaire élevé et étude du taux de récidive. Andrologie 1992, Soumis pour publication.

22 - Gordon JW, Grunfeld L, Garisi GJ, Talanski BE, Richards C, Lanfer N: Fertilization of human oocytes by sperm from infertile males after zona pellucida drilling. Fertil. Steril., 1988, 50:68-73.

23 - Gordon JW, Talansky BE: Assisted fertilization by zona drilling : a mouse model for correction of oligospermia. J. Exp. Zool., 1986, 239:347-354.

24 - Hill DL, Adler D, Rothman C, Surrey M, Danzer $\mathrm{H}$, Friedman S: Micromanipulation in a centre for reproductive medicine. Fertil. Steril,, 1991, 55:36-38.

25 - Jean M, Barriere P, Sagot P: L'Hermite A. Lopes P. : Utility of zona pellucida drilling in cases of severe semen alterations in man. Fertil. Steril., 1992, 57:591596.

26 - Jean M, L'Hermite A, Barriere P: Intérêt du "zona drilling" dans les tests de fécondation pré-IAD. Contr. Fertil. Sex., 1990, 18:570-571.

27 - Johnson MH, Vincent C, Pickering SJ, Cant A, Bruade PR, Currie J: Acid tyrode's solution can stimulate pathogenetic activation of human and mouse oocytes. Fertil. Steril, 1990, 53:266-270.

28 - Khalifa E, Oehninger S, Acosta AA, Morshedi M, Veeck L, Bryzyski RG, Muasher SJ: Successful fertilization and pregnancy outcome in in-vitro fertilization using cryopreserved/thawed spermatozoa from patients with malignant diseases. Hum. Reprod,. 1992, 7:105108.

29 - Kola I, Lachnam O, Jansen RPS, Turner M, Trounson A: Chromosome analysis of human oocytes fertilized by microinjection of spermatozoa into the perivitelline space. Human Reprod., 1990, 5:575-577.

30 - Kruger TF, Acosta AA, Simmons KF, Swanson RJ, Matta JF, Oehninger S : Predictive value of abnormal sperm morphology in in vitro fertilization. Fertil. Steril., 1988, 49:112-117.

31 - Lanzendorf S, Maloney MK, Veeck LL, Slusser J, Hodgen GD, Rosenwaks Z: A preclinical evaluation of pronuclear formation by microinjection of human spermatozoa into human oocytes. Fertil. Steril ; 1988, 49:835-842.

32 - Lassalle B, Courtot AM, Testart J: In vitro fertilization of hamster and human oocytes by microinjection of human sperm. Gamete Res., 1987, 16:69-78.

33 - Laufer N, Abrams T, Simon A: Treatment of male infertility by gamete micromanipulation. Focus in Reproduction, 1991, 1: 5-9.

34 - Laws-King A, Trounson A, Sathananthan M, Kola I : Fertilization of human oocytes by microinjection of a single spermatozoon under the zona pellucida. Fertil. Steril ; 1987, 48:637-642.
35 - Lee MA, Trucco GS, Bechtol KB, Wummer N, Kopf GS, Blasco L, Storey BT: Capacitation and acrosomic reactions in human spermatozoa monitored by a chlortetracycline fluorescence assay. Fertil. Steril, 1987, 48:649-658.

36 - Mahadevan MM, Trounson AO: Removal of the cumulus oophorus from the human oocyte for in vitro fertilization. Fertil. Steril., 1985, 43:263-267.

37 - Malter HE, Cohen J: Partial zona dissection of the human oocyte : a non traumatic method using micromanipulation to assist zona pellucida penetration. Fertil. Steril, 1989, 51:139-148.

38 - Malter HE, Cohen J: Embryonic development after microsurgical repair of polyspermic human zygotes. Fertil. Steril., 1989, 52:373-382.

39 - Malter HE, Talansky B, Gordon J, Cohen J: Monospermy and polyspermy after partial zona dissection of reinseminated human oocytes. Gamete Res. 1989, 23:377-386.

40 - Markert CL: Fertilization of mammalian eggs by sperm injection. J. Exp. Zool., 1983, 228: 195-201.

41 - Mortimer D, Chorney MJ, Curtis EF, Trounson $\mathrm{AD}$ : Calcium dependence of human sperm fertilizing ability. J. Exp. Zool., 1988, 246:194-201.

42 - Ng SC, Bongso A, Chang SI, Sathananthan H, Ratnam S: Transfer of human sperm into the perivitelline space of human oocytes after zona-drilling or zona-puncture. Fertil. Steril., 1989, 52:73-78.

43 - Ng SC, Bongso A, Ratman SS: Microinjection of human oocytes : a technique for severe oligoasthenoteratospermia. Fertil. Steril, 1991, $56: 117-1123$.

44 - Ng SC, Bongso A, Ratnam SS, Sathonanthan H, Chan CLK, Wong PC, Hagglund L, Anandakumar C, Wong YC, Goh VHH: Pregnancy after transfer of sperm under zona. Lancet., 1988, 2:790.

45 - Ng SC, Bongso A, Sathananthan AH, Ratnam SS Micromanipulation: its relevance to human in vitro fertilization. Fertil, Steril, 1990, 53:203-219.

46 - Olar TT, Lanasa J, Dickeky RT, Taylor SN, Curolz DN: Fertilization of human oocytes by microinjection of human sperm aspirated from the caput epididymis of an individual with obstructive azoospermia. J. In vitro Fert. Embryo. Transfer., 1990, 7:160164.

47 - Palanker D, Ohad S, Lewis A, Simon A, Shenkar J, Penchas S, Laufer N: Technique for cellular microsurgery using the 193-nm excimer laser. Lasers in Surgery and Medicine,. 1991, 11:580-586.

48 - Palermo G, Joris H, Devroey P, Van Steirteghem AC: Induction of acrosome reaction in human spermatozoa used for subzonal insemination. Human Reprod, 1992, 7:248-254.

49 - Palermo G, Joris H, Devroey P, Van Steirteghem A: Pregnancies after intracytoplasmic injection of single spermatozoon into an oocyte. The Lancet, 1992, 340:17-18

50 - Payne D, McLaughlin KJ, Depypere HT, Kirby CA, Warnes GM, Matthews CD: Experience with zona cutting to improve fertilization rates of human oocytes in vitro. Human Reproduction,. 1991, 6:423-431. 
5l - Ron-El R, Nachum A, Herman A, Golan A, Caspi E, Soffer Y: Delayed fertilization and poor embryonic development associated with impaired semen quality. Fertil. Steril, 1991, 55:338-344.

52 - Sakkas D, Lacham 0, Ganaroli L, Trounson A: Subzonal sperm microinjection in cases of severe male factor infertility and repeated in vitro fertilization failure. Fertil. Steril, 1992, 57:1279-1288.

53 - Sathananthan AH, Chen C: Sperm-oocyte membrane fusion in the human during monospermic fertilization. Gamete Res., 1986, 15:177-186.

54 - Sathananthan AH, Ng SC, Edirisinghe WR, Ratnam SS, Wong PC: Sperm-oocyte interaction in the human during polyspermic fertilization in vitro. Gamete Res., 1986, 15: 317-326.

55 - Sathananthan AH, Ng SC, Trounson A, Bongso A, Laws- King A, Ratman SS: Human microinsemination by injection of single or multiple sperm: ultrastructure. Human Reprod., 1989, 4:574-583.

56 - Sathananthan AH, Trounson AO: Ultrastructure of cortical granule release and zona interaction in monospermic and polyspermic human ova fertilized in vitro. Gamete Res., 1982, 6: 225-234.

57 - Siiteri JE, Dandekar P, Meizel S: Human sperm acrosome reaction-initiating activity associated with the human cumulus oophorus and mural granulosa cells. J. Exp. Zool., 1986. 246: $71-80$.
58 - Simon A, Younis J, Lewin A, Bartoov B, Schenker JG, Laufer N: The correlation between sperm cell morphology and fertilization after zona pellucida slitting in subfertile males. Fertil. Steril, 1991 , $56: 325-331$.

59 - Stock CE, Bates R, Lindsay KS, Edmonds DK, Fraser LR: Human oocyte cumulus complexes stimulate the human acrosome reaction. J. Reprod. Fertil., $1989,86: 723-730$.

60 - Tadir Y, Wright WH, Vafa 0, Liaw LH, Asch R, Berns MW: Micromanipulation of gametes using laser microbeams. Human Reprod., 1991, 7: 1011 -1016.

61 - Tucker MJ, Bishop FM, Cohen J, Weker SR, Wright G: Routine application of partial zona dissection for male factor infertility. Human Reprod., 1991, 6: 676-681.

62 - Van Der Zwalmen P, Barlow P, Nijs M, Bertin G, Leroy F, Schoysman R: Usefulness of partial zona dissection of the zona pellucida in an in vitro fertilization programme. Human Reprod., 1992, 7: 537-544.

63 - Veeck LL, Oehninger S, Acosta AA, Muasher SJ: Sperm microinjection in a clinical in vitro fertilization program. 45th annual meeting of the AFS. Abstract O121. Fertil. Steril, Suppl 1989, pp S50-S51.

64 - Wittemer C, Moreau L, Dellenbach P, Gerlinger P. Le Meur MA, Chambon P: A case of human pregnancy after microinjection of capacitated sperm into the perivitelline space. J. In vitro Fert. Embryo Transfer, 1991, 8:222-224.

65 - Wolf JP, Jouannet P: Intérêt des méthodes de microfécondation dans l'infertilité masculine. $30 \mathrm{e}$ réunion de la Société fran aise pour l'étude de la fertilité. Paris, 3-4 octobre 1991. Contr. Sex. 1991, 19: 168-176.

RESUME : Depuis quelques années, on assiste, dans le domaine des technologies médicalement assistées, à un engouement pour l'utilisation des micromanipulations comme thérapeutique de l'infertilité masculine. Une revue de la littérature nous a permis de constater qu'à ce jour moins de 100 grossesses ont été obtenues et que les taux de fécondation sont trés variables en fonction de la rigueur de la sélection des cas. Alors que la dissection partielle de la zone pellucide ne semble pas être un technique prometteuse, l'injection de spermatozoides dans l'espace périvitellin ou dans le cytoplasme de l'ovocyte pourrait être promise à un bel avenir. Mots clés : Micromanipulation, Microinjection, PZD, SUZI, ICSI, FIVETE. Andrologie, 1992, 2 : 61-67. 